Supporting information

\title{
High-Performance Organic Solar Cells Featuring Double Bulk Heterojunction Structures with Vertical- Gradient Selenium Heterocyclic Nonfullerene Acceptor Concentrations
}

Hao-Wen Cheng, Anisha Mohapatra, Yi-Ming Chang, Chuang-Yi Liao, Yu-Tang Hsiao, ChungHao Chen, Yu-Che Lin, Shih-Yu Huang, Bin Chang, Yang Yang, Chih-Wei Chu*, and Kung-Hwa $W e i^{*}$

[*] H.-W. Cheng, C.-H. Chen, Y.-C. Lin, S.-Y. Huang, B. Chang, K.-H. Wei

Department of Materials Science and Engineering, Center for Emergent Functional Matter Science

National Yang Ming Chiao Tung University, Hsinchu 3001, Taiwan

E-mail: khwei@mail.nctu.edu.tw

H.-W. Cheng, C.-H. Chen, Y.-C. Lin, S.-Y. Huang, B. Chang, K.-H. Wei

Department of Materials Science and Engineering, Center for Emergent Functional Matter Science

National Chiao Tung University, Hsinchu 3001, Taiwan

E-mail: khwei@mail.nctu.edu.tw

[*] A. Mohapatra, C.W. Chu

Research Center for Applied Science 
Academia Sinica

Taipei, 115, Taiwan

Email: gchu@gate.sinica.edu.tw

[*] C.W. Chu

College of Engineering, Green Technology Research Center

Chang Gung University

Taoyuan City, 33302, Taiwan (R.O.C.)

Email: gchu@gate.sinica.edu.tw

Y.-M. Chang, C.-Y. Liao, Y.-T. Hsiao

Raynergy Tek Incorporation, 2F, 60, Park Ave. 2

Hsinchu Science Park

Hsinchu 30844, Taiwan

Y. Yang

Department of Materials Science and Engineering, California NanoSystems Institute University of California

Los Angeles, CA 90095, USA 


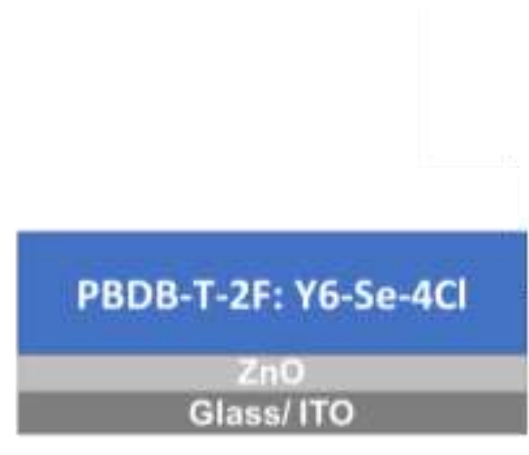

Spin-coated first BHJ film

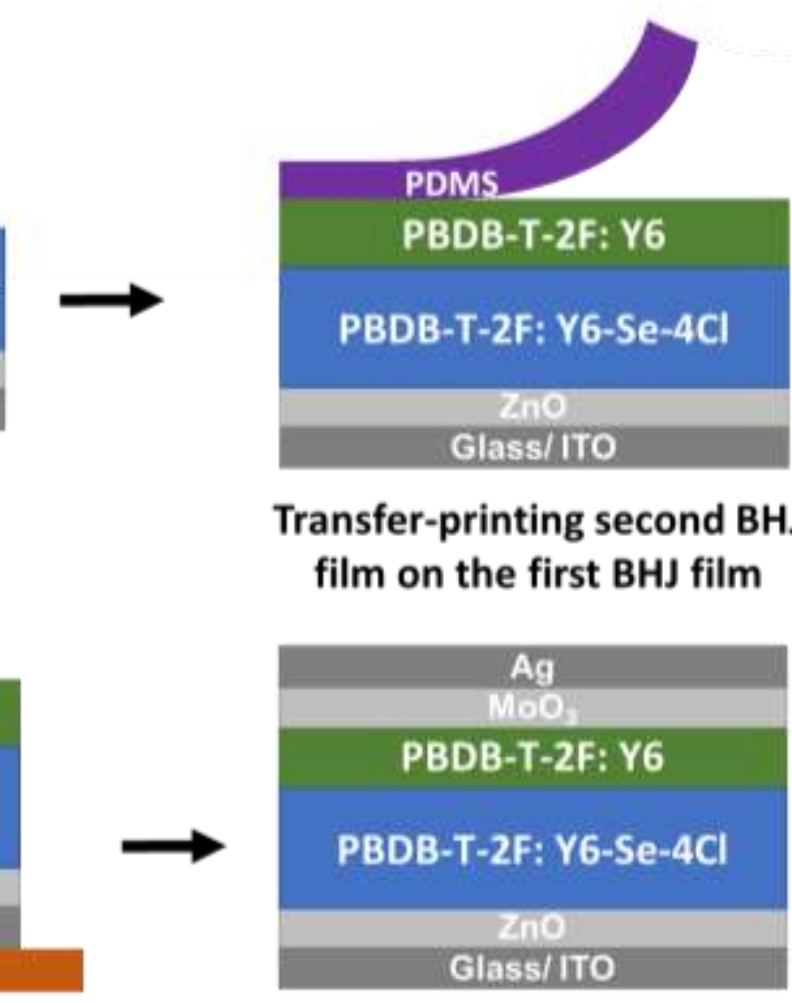

Thermal annealing

Figure S1. The scheme of the process of preparing the double-BHJ structure devices via transferprinting process. 
a)

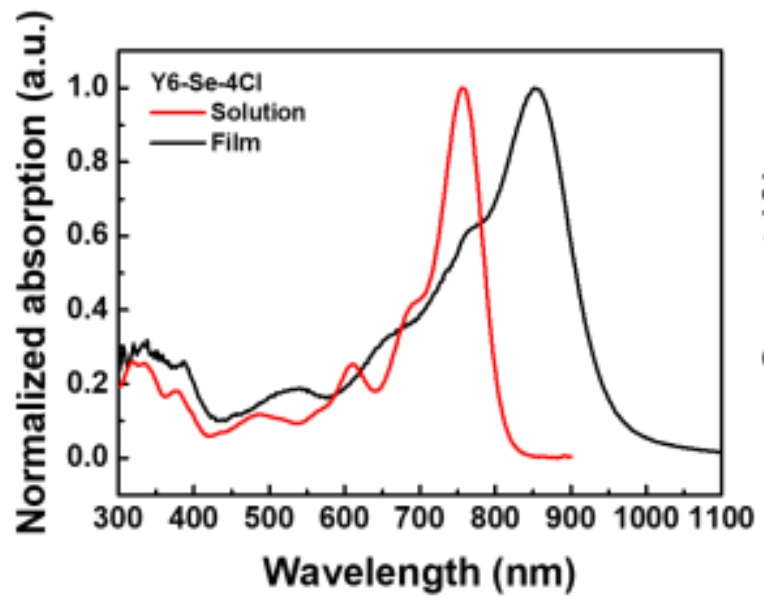

b)

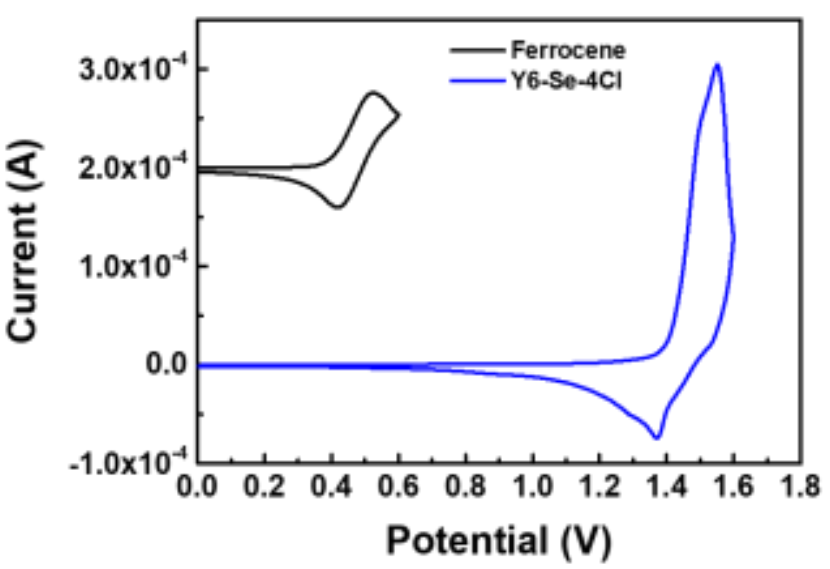

Figure S2. a) The normalized absorption of $\mathrm{Y} 6-\mathrm{Se}-4 \mathrm{Cl}$ in film and solution. b) The cyclic voltammetry curve of Y6-Se-4Cl. 


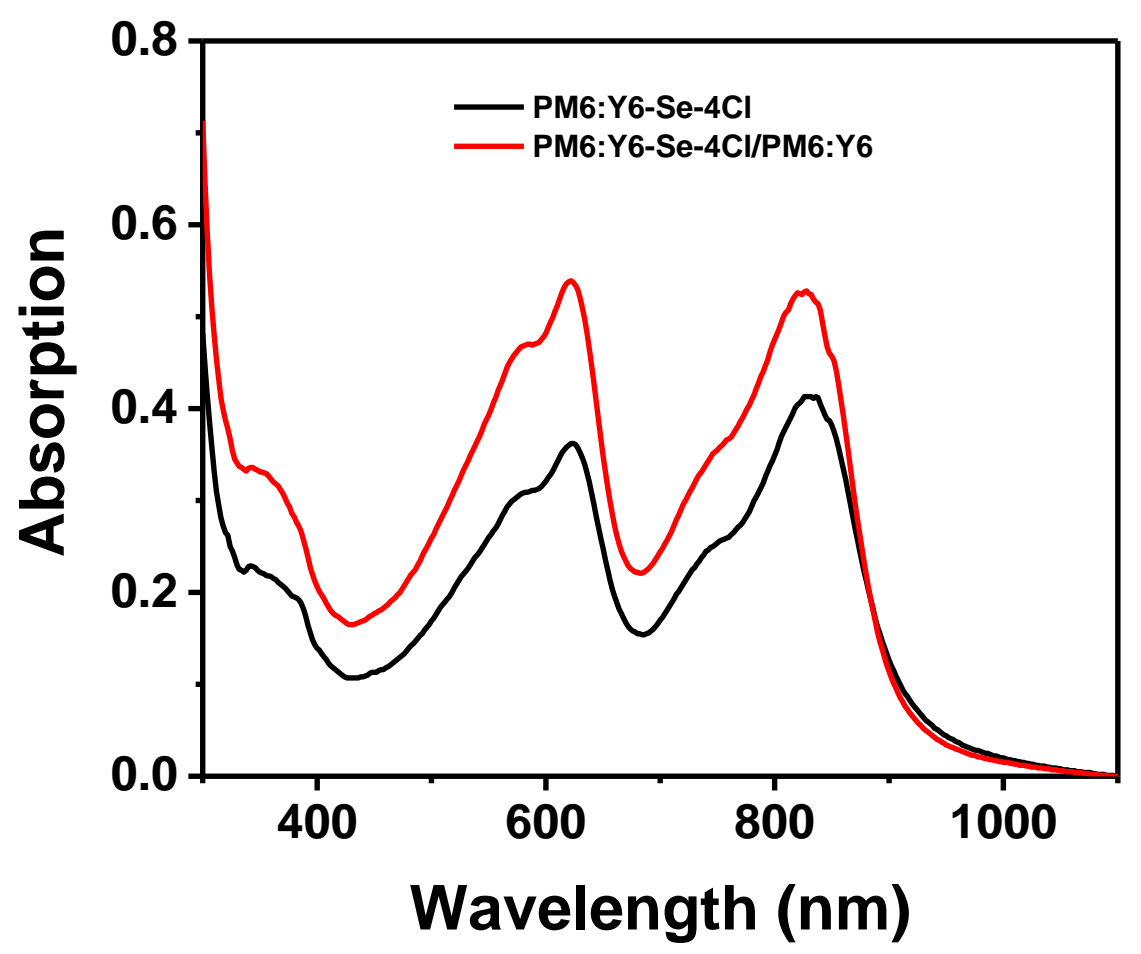

Figure S3. The absorption spectrum of PM6:Y6-Se-4Cl (one-BHJ structure) and PM6:Y6-Se4C1/PM6:Y6 (double-BHJ structure) films. 
a)

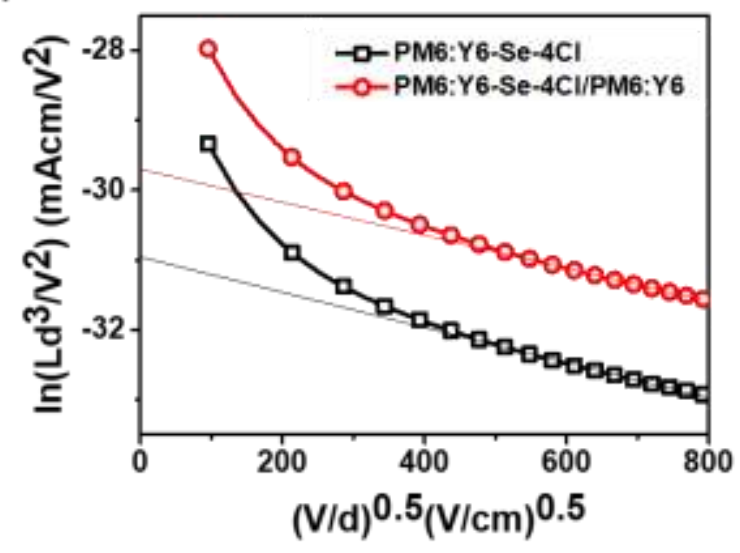

b)

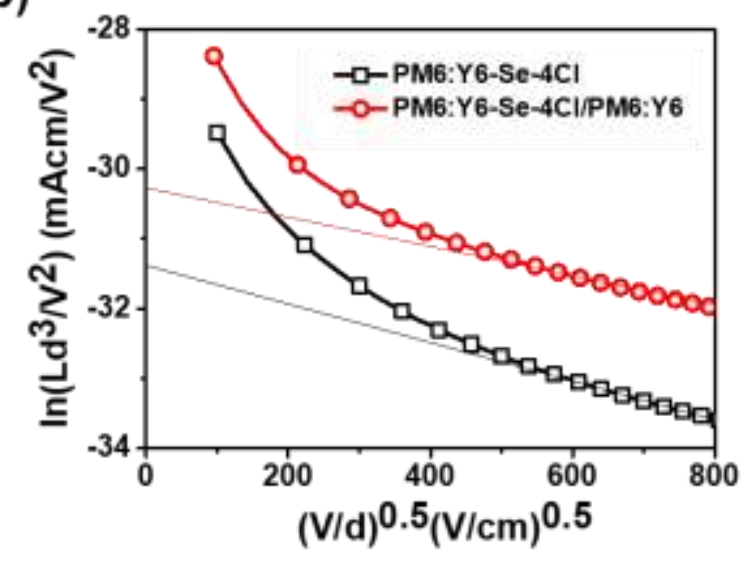

Figure S4 a) and b) Hole-only and electron-only based on the PM6:Y6-Se-4Cl (one-BHJ structure) and PM6:Y6-Se-4Cl/ PM6:Y6 (double-BHJ structure) devices. 
a)

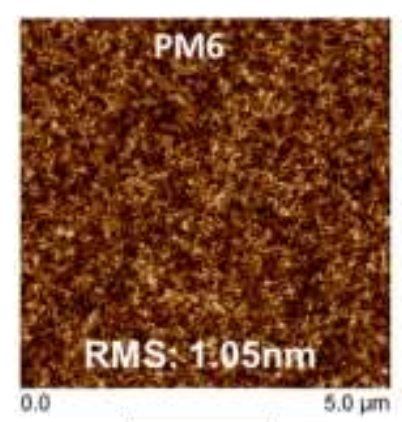

c)

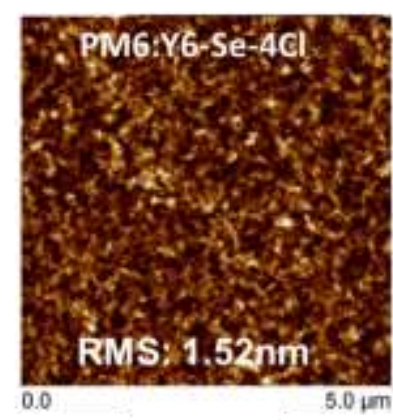

b)

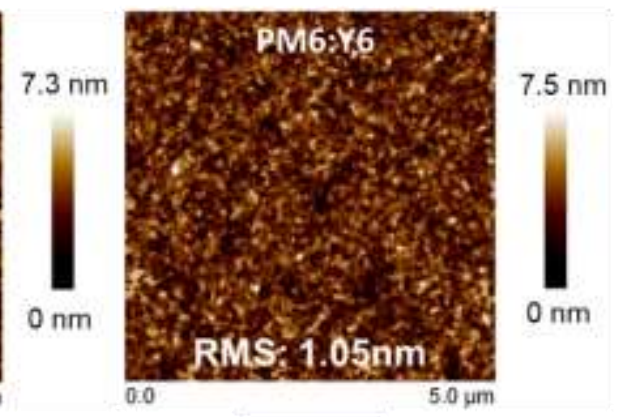

d)

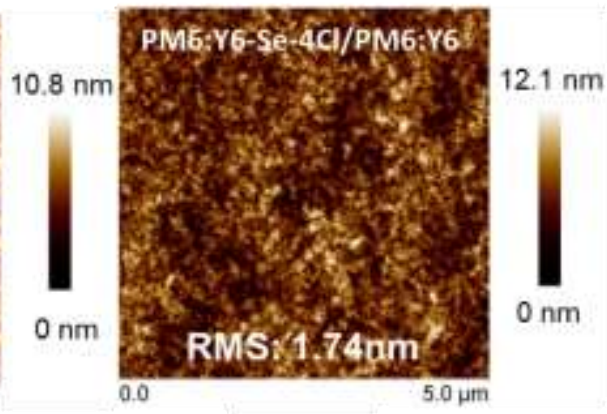

Figure S5. a-d) AFM height images ( 5 x $5 \mu \mathrm{m}$ ) of films of pure PM6, PM6:Y6, PM6:Y6-Se-4Cl and PM6:Y6-Se-4Cl/ PM6:Y6 films. 


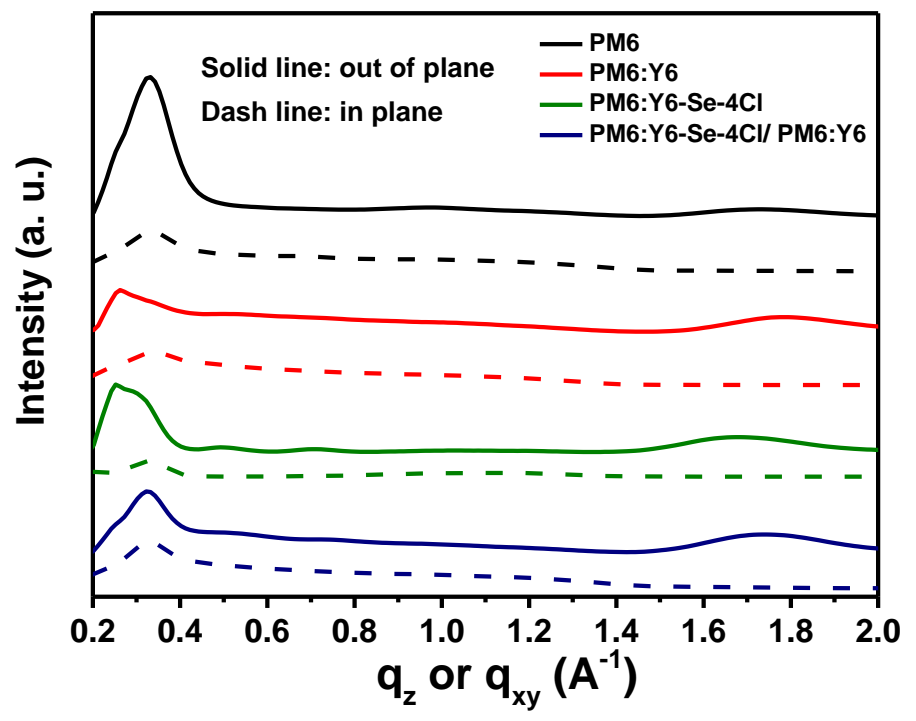

Figure S6. The one-dimensional GIWAX patterns of pure PM6, PM6:Y6, PM6:Y6-Se-4Cl and PM6:Y6-Se-4Cl/ PM6:Y6 films. 
a)

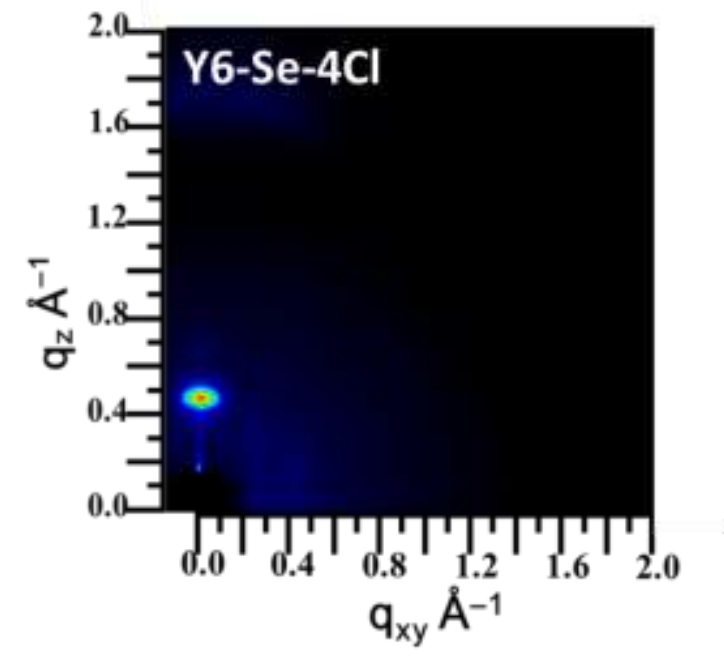

b)

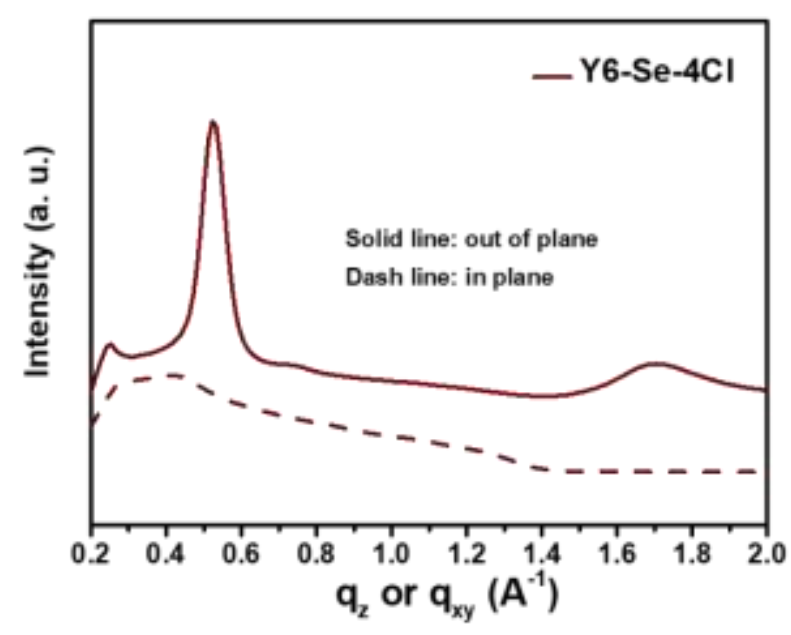

Figure S7. a) 2D and b) 1D GIWAX patterns of pure $\mathrm{Y} 6-\mathrm{Se}-4 \mathrm{Cl}$ film. 
Table S1. Photovoltaic performance of the champion devices based on PM6:Y6-Se-4Cl, PM6:Y6, PM6:IT-4Cl, PM6:Y6-Se-4Cl/PM6:Y6, and PM6:Y6-Se-4Cl/PM6:IT-4Cl.

\begin{tabular}{|c|c|c|c|c|c|}
\hline & $\begin{array}{l}\text { Active layer } \\
\text { structure }\end{array}$ & $\operatorname{Voc}(\mathbf{V})$ & $J_{\mathrm{SC}}\left(\mathbf{m A ~} \mathbf{c m}^{-2}\right)$ & $\begin{array}{l}\mathbf{F F} \\
(\%)\end{array}$ & $\begin{array}{c}\text { Champion PCE } \\
(\%)\end{array}$ \\
\hline PM6:Y6-Se-4Cl (120 nm) & Traditional & 0.84 & 26.4 & 67.7 & 15.0 \\
\hline PM6:Y6 (100 nm) & Traditional & 0.84 & 25.9 & 70.6 & 15.4 \\
\hline PM6:IT-4Cl (100 nm) & Traditional & 0.80 & 21.4 & 67.6 & 11.6 \\
\hline $\begin{array}{l}\text { PM6:Y6-Se-4Cl }(100 \mathrm{~nm}) \\
\text { /PM6:Y6 }(20 \mathrm{~nm})\end{array}$ & Double-BHJ & 0.84 & 26.6 & 73.3 & 16.4 \\
\hline $\begin{array}{l}\text { PM6:Y6-Se-4Cl (100 nm) } \\
\text { /PM6:IT-4Cl }(20 \mathrm{~nm})\end{array}$ & Double-BHJ & 0.83 & 27.6 & 68.8 & 15.8 \\
\hline
\end{tabular}


Table S2. The optimization of the photovoltaic performance for the devices based on double-BHJ devices (PM6:Y6-Se-4Cl/PM6:Y6, and PM6:Y6-Se-4Cl/PM6:IT-4Cl) with different thickness of the second BHJ layer.

\begin{tabular}{|c|c|c|c|c|c|}
\hline First BHJ & Second BHJ & $V_{\text {oC }}(\mathbf{V})$ & $J_{\text {SC }}(\mathbf{m A} / \mathbf{c m})$ & FF (\%) & PCE (\%) \\
\hline PM6:Y6-Se-4Cl (100nm) & None & $0.84 \pm 0.01$ & $25.1 \pm 0.2$ & $66.4 \pm 0.2$ & $14.0 \pm 0.3$ \\
\hline PM6:Y6-Se-4Cl (100nm) & PM6:Y6 (10 nm) & $0.84 \pm 0.01$ & $26.3 \pm 0.4$ & $70.4 \pm 0.2$ & $15.6 \pm 0.3$ \\
\hline PM6:Y6-Se-4Cl (100nm) & PM6:Y6 (20 nm) & $0.84 \pm 0.01$ & $26.3 \pm 0.2$ & $73.0 \pm 0.2$ & $16.1 \pm 0.2$ \\
\hline PM6:Y6-Se-4Cl (100nm) & PM6:Y6 (30 nm) & $0.84 \pm 0.01$ & $27.3 \pm 0.4$ & $68.0 \pm 0.3$ & $15.6 \pm 0.1$ \\
\hline PM6:Y6-Se-4Cl (100nm) & PM6:IT-4Cl (10 nm) & $0.83 \pm 0.01$ & $26.4 \pm 0.2$ & $67.9 \pm 0.3$ & $14.9 \pm 0.3$ \\
\hline PM6:Y6-Se-4Cl (100nm) & PM6:IT-4Cl (20 nm) & $0.83 \pm 0.01$ & $27.4 \pm 0.3$ & $68.5 \pm 0.1$ & $15.5 \pm 0.2$ \\
\hline PM6:Y6-Se-4Cl (100nm) & PM6:IT-4Cl (30 nm) & $0.83 \pm 0.01$ & $27.6 \pm 0.3$ & $63.8 \pm 0.1$ & $14.6 \pm 0.2$ \\
\hline
\end{tabular}


Table S3. Photovoltaic performance of the PM6:Y6-Se-4Cl:Y6 based ternary-blend devices.

\begin{tabular}{|c|c|c|c|c|}
\hline Active layer (ratio, wt\%) & $V_{\text {OC }}(\mathbf{V})$ & $J_{\mathrm{SC}}\left(\mathbf{m A} \mathbf{A} \mathbf{c m}^{2}\right)$ & FF $(\%)$ & PCE $(\%)$ \\
\hline PM6:Y6-Se-4Cl (1:1.2, $\left.20 \mathrm{mg} \mathrm{mL}^{-1}\right)$ & $0.84 \pm 0.01$ & $25.9 \pm 0.3$ & $67.2 \pm 0.3$ & $14.6 \pm 0.2$ \\
\hline PM6:Y6-Se-4Cl:Y6 (1:1.2:0.1, $\left.20 \mathrm{mg} \mathrm{mL}^{-1}\right)$ & $0.83 \pm 0.01$ & $26.3 \pm 0.3$ & $69.6 \pm 0.2$ & $15.2 \pm 0.3$ \\
\hline PM6:Y6-Se-4Cl:Y6 (1:1.2:0.2, $\left.20 \mathrm{mg} \mathrm{mL}^{-1}\right)$ & $0.82 \pm 0.01$ & $27.1 \pm 0.3$ & $63.8 \pm 0.3$ & $14.2 \pm 0.3$ \\
\hline PM6:Y6-Se-4Cl:Y6 (1:1.2:0.3, $\left.20 \mathrm{mg} \mathrm{mL}^{-1}\right)$ & $0.82 \pm 0.01$ & $26.8 \pm 0.2$ & $61.8 \pm 0.3$ & $13.6 \pm 0.1$ \\
\hline PM6:Y6-Se-4Cl:Y6 (1:1.2:0.4, $\left.20 \mathrm{mg} \mathrm{mL}^{-1}\right)$ & $0.82 \pm 0.01$ & $26.2 \pm 0.2$ & $59.7 \pm 0.2$ & $12.8 \pm 0.2$ \\
\hline
\end{tabular}




\section{Synthesis route of $\mathrm{Y} 6-\mathrm{Se}-4 \mathrm{Cl}$}
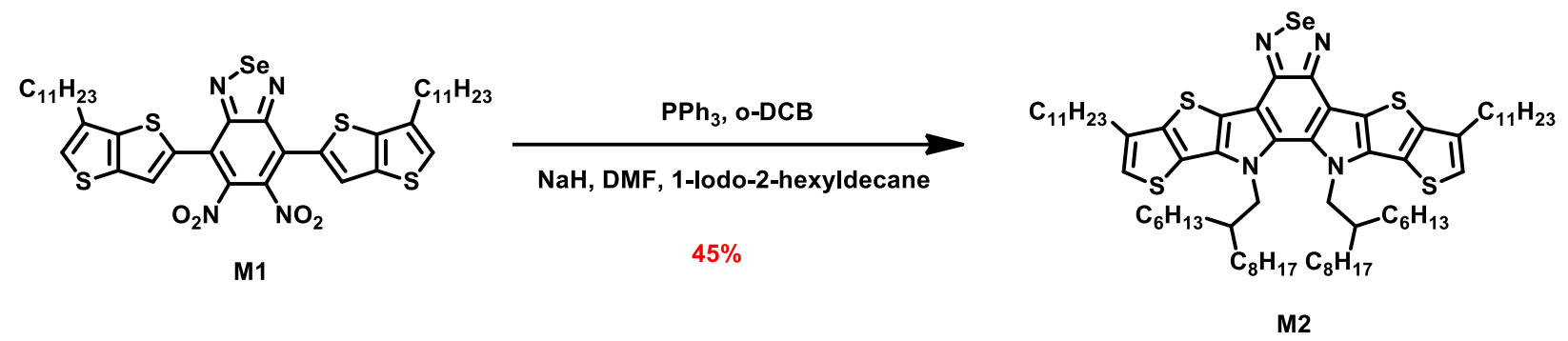

A mixture of M1 (6 g, $8.0 \mathrm{mmol})$, triphenylphosphine (21 g, $80.4 \mathrm{mmol})$ and 1,2-dichlorobenzene $(173 \mathrm{~mL})$ was degassed for 30 minutes and refluxed for 22 hours. After cooling to room temperature, methanol was added and the mixture was filtered under reduced pressure. The residue was purified by column chromatography (heptane/dichloromethane, v/v, 1/1) to give an orange solid. Subsequently, the orange intermediate was then mixed with $\mathrm{NaH}(60 \%$ in oil, $2.1 \mathrm{~g}, 51.7$ mmol), 1-iodo-2-hexyldecane (20.5 g, $58.2 \mathrm{mmol})$ and anhydrous DMF (100 mL) were mixed under argon and stirred at $80^{\circ} \mathrm{C}$ overnight. The mixture was extracted with ethyl acetate for three times. The combined organic phase was washed with water followed by brine. Then, the solution was dried over $\mathrm{Mg}_{2} \mathrm{SO}_{4}$ and concentrated under reduced pressure. The residue was purified by column chromatography (heptane/dichloromethane, v/v, 1/1), to give an orange solid $3.6 \mathrm{~g}, 45 \%$ yield, two steps). ${ }^{1} \mathrm{H}$ NMR (500 MHz, $\left.\mathrm{CDCl}_{3}\right): \delta 6.97(\mathrm{~s}, 2 \mathrm{H}), 4.52(\mathrm{~d}, J=8.0 \mathrm{~Hz}, 4 \mathrm{H}), 2.79$ (t, $J=7.8 \mathrm{~Hz}, 4 \mathrm{H}), 2.09-2.04(\mathrm{~m}, 2 \mathrm{H}), 1.83(\mathrm{p}, J=7.6 \mathrm{~Hz}, 4 \mathrm{H}), 1.45-0.79(\mathrm{~m}, 92 \mathrm{H}), 0.66(\mathrm{~d}, J=7.0 \mathrm{~Hz}$ $6 \mathrm{H}) \circ$
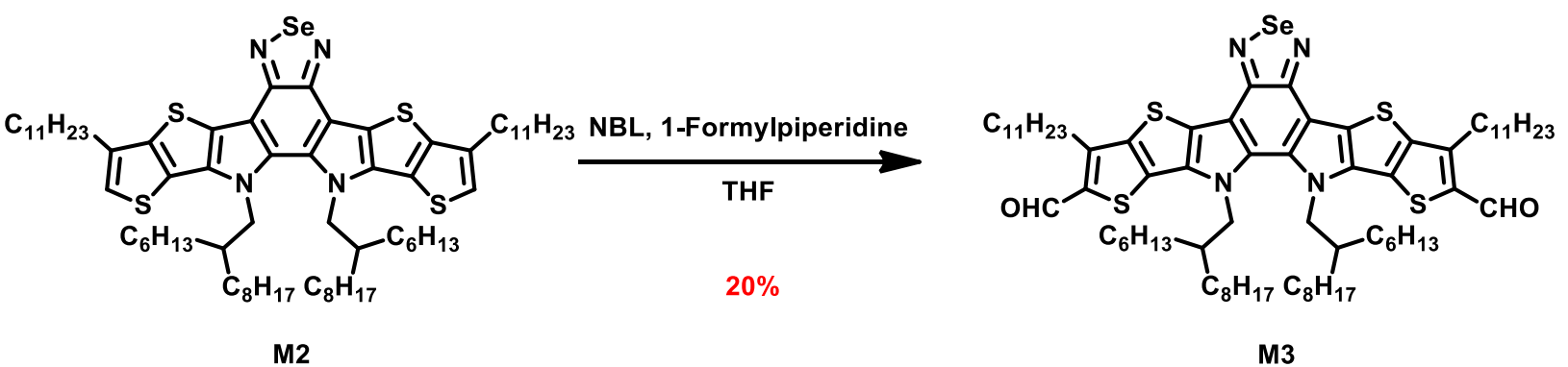

M3 
A mixture of compound M2 (800 mg, $0.64 \mathrm{mmol})$ and anhydrous THF (32 mL), $2.5 \mathrm{M}$-butyl lithium $(0.6 \mathrm{~mL}, 1.4 \mathrm{mmol})$ was added dropwise slowly at $-78{ }^{\circ} \mathrm{C}$ under argon. The mixture was stirred at $-78{ }^{\circ} \mathrm{C}$ for 30 minutes, and then anhydrous 1 -formylpiperidine $(0.71 \mathrm{ml}, 6.4 \mathrm{mmol})$ was added. The mixture was stirred 30 minutes at room temperature. Brine was added and the mixture was extracted with ethyl acetate for three times. The combined organic phase was washed with water followed by brine. Then the solution was dried over $\mathrm{Mg}_{2} \mathrm{SO}_{4}$ and concentrated under reduced pressure. The residue was purified by column chromatography (heptane/dichloromethane, 1/1, v/v) to get the product as orange solid $(157 \mathrm{mg}, 20 \%) .{ }^{1} \mathrm{H} \mathrm{NMR}\left(500 \mathrm{MHz}, \mathrm{CDCl}_{3}\right): \delta 10.11(\mathrm{~s}, 2 \mathrm{H})$, $4.55(\mathrm{~d}, J=8.0 \mathrm{~Hz}, 4 \mathrm{H}), 3.17$ (t, J=7.8 Hz, 4H), 2.04-2.01 (m, 2H), 1.90 (p, J=7.6 Hz, 4H), 1.48$0.78(\mathrm{~m}, 92 \mathrm{H}), 0.66(\mathrm{~d}, J=7.0 \mathrm{~Hz}, 6 \mathrm{H}) \circ$

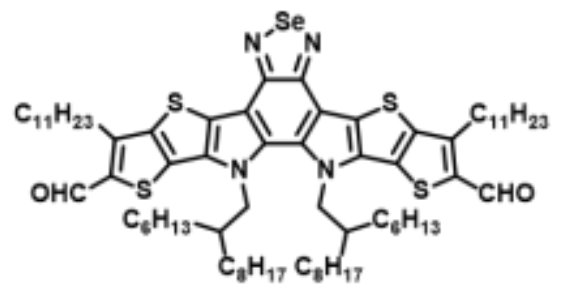

M3

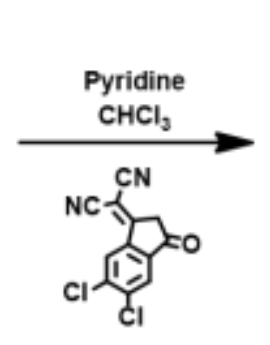

$60 \%$

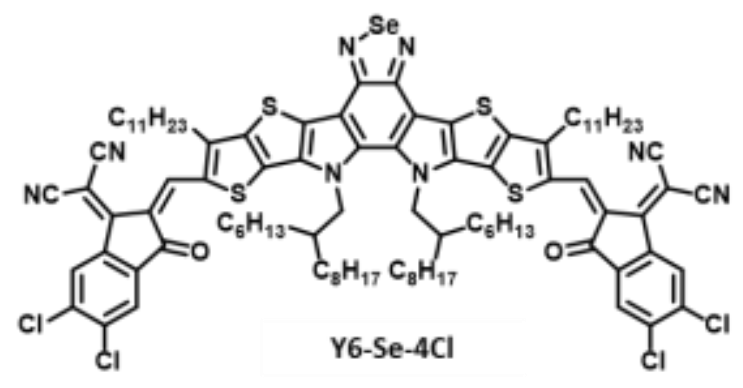

A mixture of compound M3 $(510 \mathrm{mg}, 0.39 \mathrm{mmol})$ and 2-(5,6-dichloro-3-oxo-2,3-dihydro1Hinden-1-ylidene)malononitrile $(515 \mathrm{mg}, 1.96 \mathrm{mmol})$ in chloroform $(15 \mathrm{~mL})$ was degassed before pyridine $(0.5 \mathrm{~mL})$ was added. The reaction was kept at $65^{\circ} \mathrm{C}$ under argon for 30 minutes. The solvent was removed under reduced pressure and the residue was washed by methanol and acetone to get the product as dark blue solid (421 mg, 60\%). ${ }^{1} \mathrm{H}$ NMR $\left(500 \mathrm{MHz}, \mathrm{CDCl}_{3}\right): \delta 9.15(\mathrm{~s}, 2 \mathrm{H})$, $8.77(\mathrm{~s}, 4 \mathrm{H}), 7.93(\mathrm{~s}, 2 \mathrm{H}), 4.70(\mathrm{~d}, J=7.5 \mathrm{~Hz}, 4 \mathrm{H}), 3.20(\mathrm{t}, J=8 \mathrm{~Hz}, 4 \mathrm{H}), 2.14-2.09$ (m, 2H), 1.85 (p, $J=7.8 \mathrm{~Hz}, 4 \mathrm{H}), 1.51-0.75(\mathrm{~m}, 92 \mathrm{H}), 0.67(\mathrm{~d}, J=6.8 \mathrm{~Hz}, 6 \mathrm{H}) \circ$ 
NMR

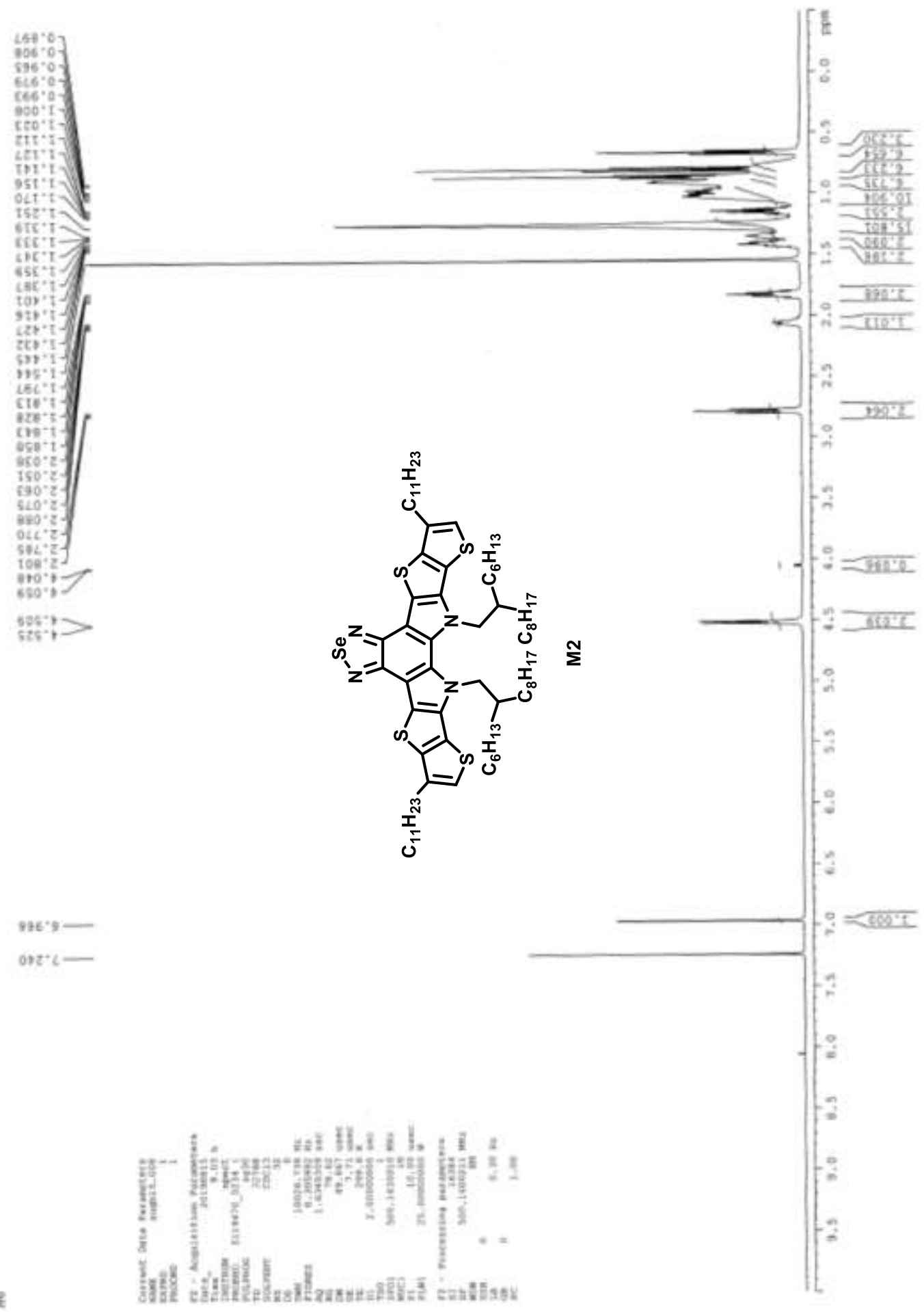




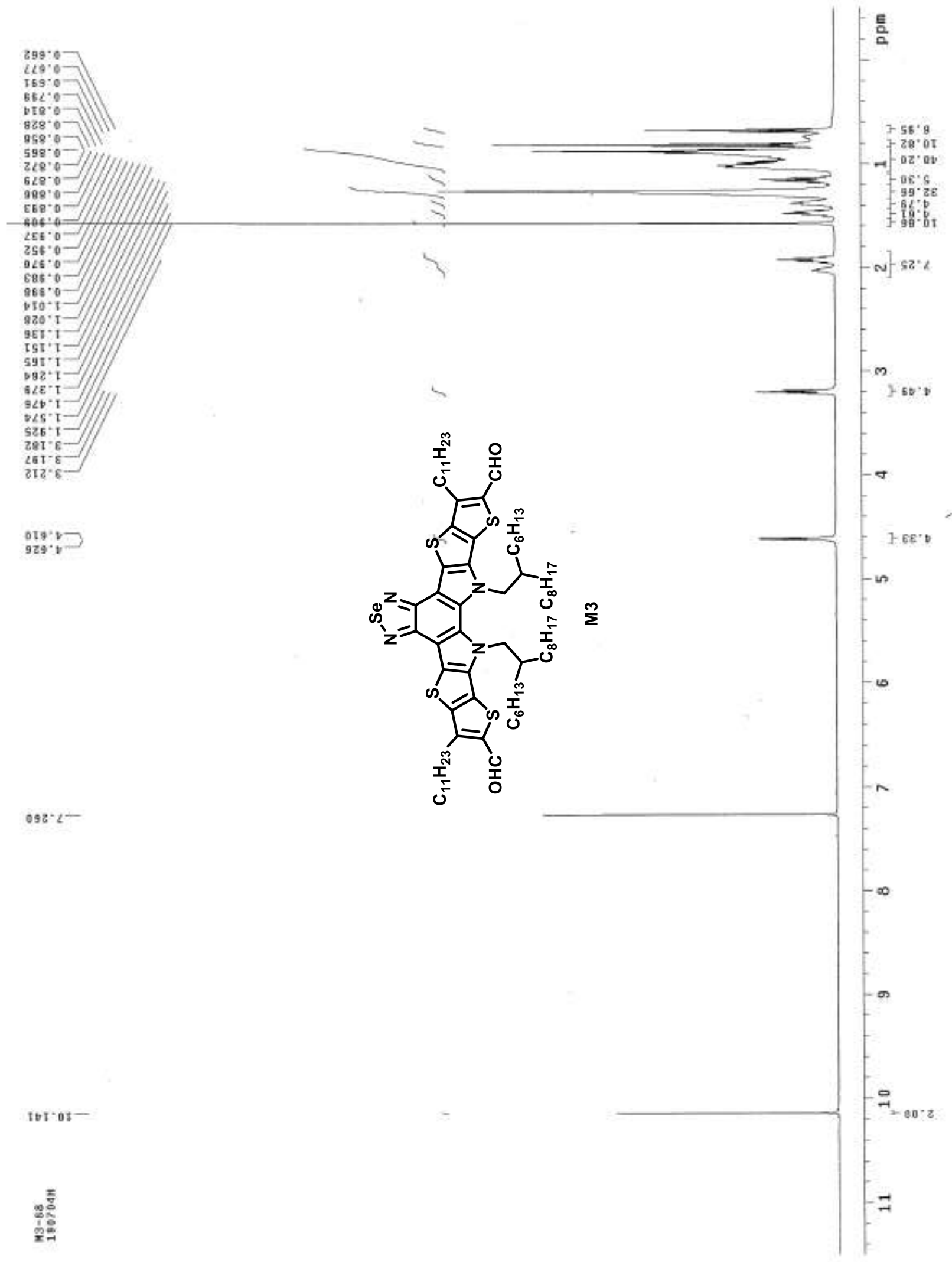




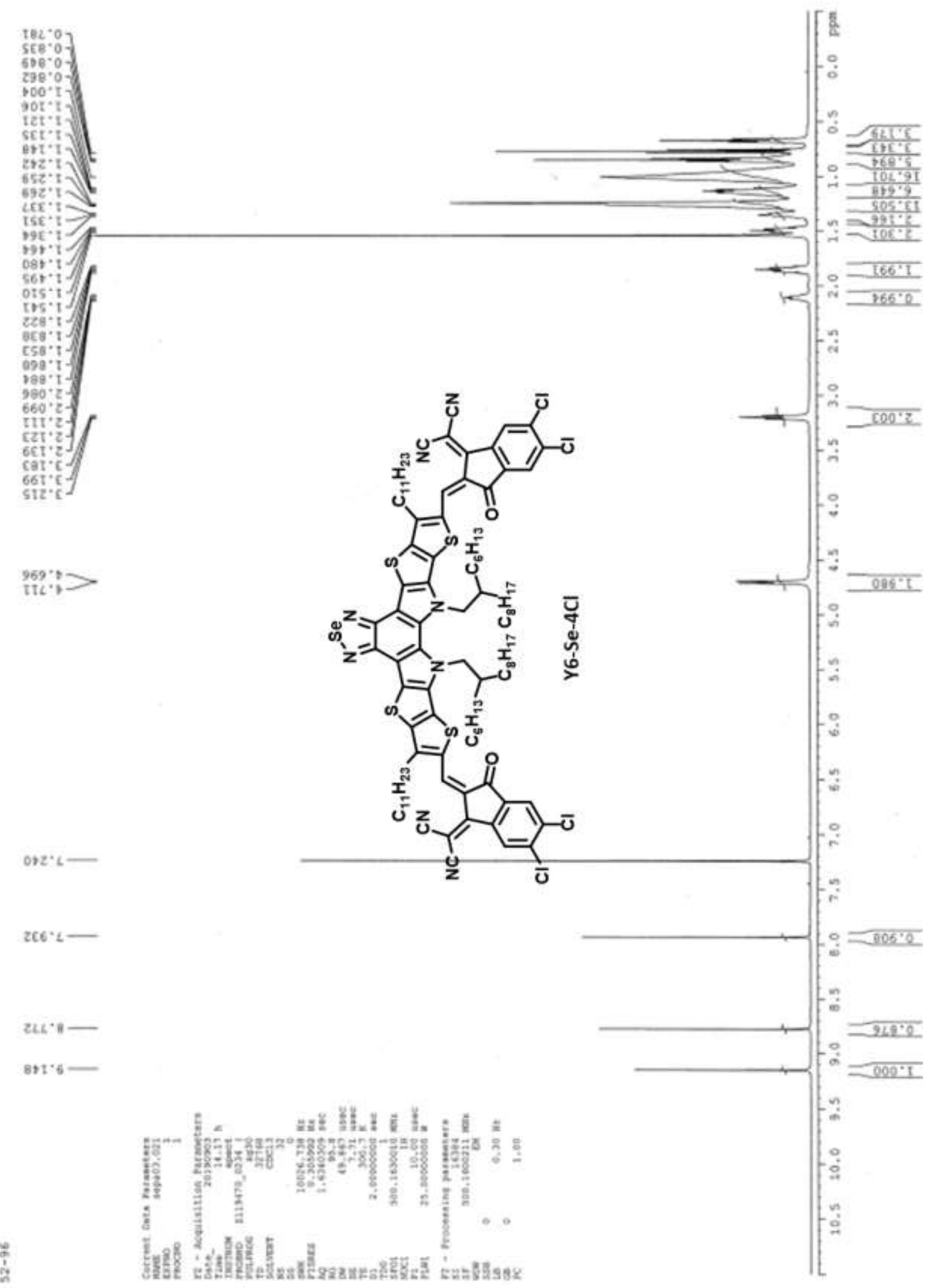

